\title{
Image Guidance of Intracardiac Ultrasound with Fusion of Pre-operative Images ${ }^{\star}$
}

\author{
Yiyong Sun ${ }^{1}$, Samuel Kadoury ${ }^{1}$, Yong $\mathrm{Li}^{1}$, Matthias John ${ }^{2}$, Jeff Resnick ${ }^{3}$, \\ Gerry Plambeck ${ }^{3}$, Rui Liao ${ }^{1}$, Frank Sauer ${ }^{1}$, and Chenyang $\mathrm{Xu}^{1}$ \\ ${ }^{1}$ Siemens Corporate Research, Princeton, NJ, USA \\ yiyong. sun@siemens.com \\ ${ }^{2}$ Siemens Medical Solutions, Erlangen, Germany \\ ${ }^{3}$ Siemens Medical Solutions, Mountain View, CA, USA
}

\begin{abstract}
This paper presents a method for registering 3D intracardiac echo (ICE) to pre-operative images. A magnetic tracking sensor is integrated on the ICE catheter tip to provide the 3D location and orientation. The user guides the catheter into the patient heart to acquire a series of ultrasound images covering the anatomy of the heart chambers. An automatic intensity-based registration algorithm is applied to align these ultrasound images with pre-operative images. One of the important applications is to help electrophysiology doctors to treat complicated atrial fibrillation cases. After registration, the doctor can see the position and orientation of the ICE catheter and other tracked catheters inside the heart anatomy in real time. The image guidance provided by this technique may increase the ablation accuracy and reduce the amount of time for the electrophysiology procedures. We show successful image registration results from animal experiments.
\end{abstract}

\section{Introduction}

Atrial fibrillation (AFib) is a leading cause of stroke. About 2.2 million people in US have AFib. There are 200,000 new cases in 2006. Current pulmonary vein ablation techniques are achieving success rates of $85 \%$ in curing paroxysmal AFib with low risk [1]. During pulmonary vein ablation, a soft thin flexible tube with an electrode at the tip is inserted through a large vein or artery in the groin and moved into the heart. This catheter is directed to the locations in the heart that produce AFib to burn them off. Currently many heart centers have the cathlabs performing pulmonary vein ablation of AFib on a regular basis.

Intracardiac echo (ICE) catheters are routinely used in many cathlabs for transseptal catheterization and left atrial procedures. It offers the unique advantage to visualize anatomy and hemodynamics while also providing the interventional cardiac electrophysiologist real time feedback on other catheters deployed in the heart [2]. However, the ultrasound image generated from the ICE catheter

\footnotetext{
* We would like to thank Wolfgang Wein, Norbert Strobel, and Ann Dempsey at Siemens, Dr. Rebecca Fahrig and Dr. Amin Al-Ahmad at Stanford University Medical Center for their support on this work.
} 


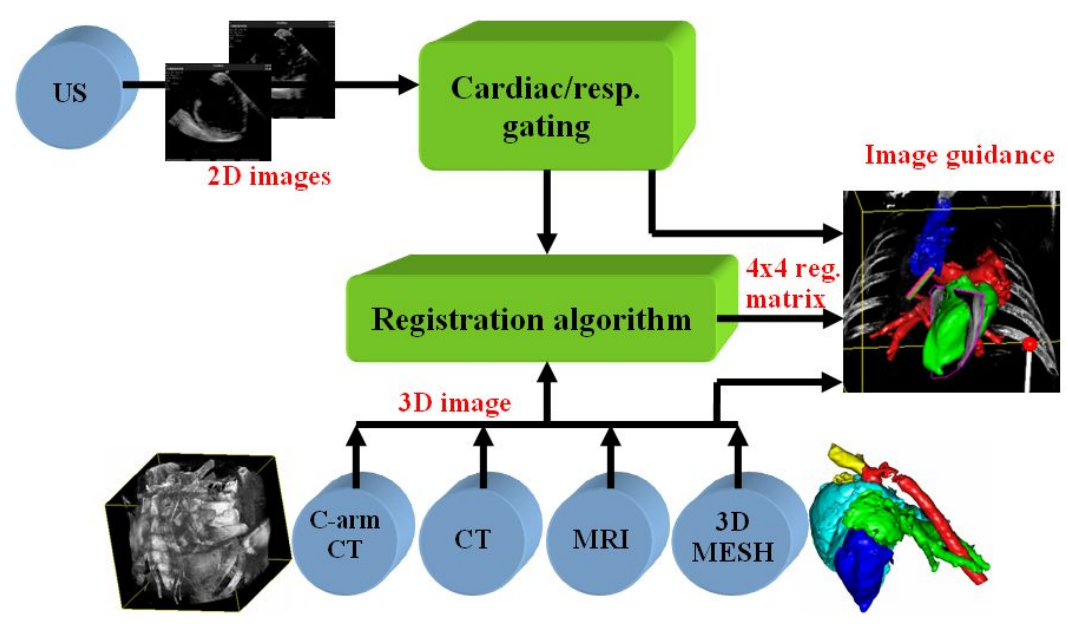

Fig. 1. System overview

has a limit field of view compared to other modalities such as fluoroscopy. Due to its flexibility, the image plane can drastically change. It requires a lot of experience and agility of the doctor to get familiar of operating the ICE catheter.

By fusing the 3D tracked ICE images with the pre-operative images such as CT, C-arm CT1 1 , or MR, doctor can see the position and orientation of the ICE catheter inside the heart in real time. Since multiple sensors can be tracked simultaneously, a tracked ablation catheter is automatically registered to the preoperative image after completing the registration of the tracked ICE catheter. The image guidance provided by this technique can increase the ablation accuracy and reduce the amount of time for the electrophysiology (EP) procedures.

Figure 1 shows the framework of registering tracked ultrasound sequence to 3D images. In this paper particularly, we register it to $\mathrm{C}$-arm CT data. The advantage of using $\mathrm{C}$-arm $\mathrm{CT}$ data is the high resolution $3 \mathrm{D}$ image can be obtained inside the EP lab immediately before or during the intervention. Our method also applies to the conventional CT and MR pre-operative data. The background is presented in Section 2. Section 3 describes the method. Experimental results are showed in Section 4, followed by discussion in Section 5 .

\section{Background}

The registration of intra-operative ultrasound images (US) and pre-operative $\mathrm{CT} / \mathrm{MR}$ images has been proposed to aid the interventional and surgical procedures. Roche et al. 4 rigidly register 3D ultrasound with MR images using bivariate correlation ratio as similarity measure. Penney et al. [5] propose to register a set of ultrasound slices and a $\mathrm{MR}$ volume of a liver by converting

${ }^{1}$ CT-like images generated by $\mathrm{C}$-arm system in the operating room 3 . 


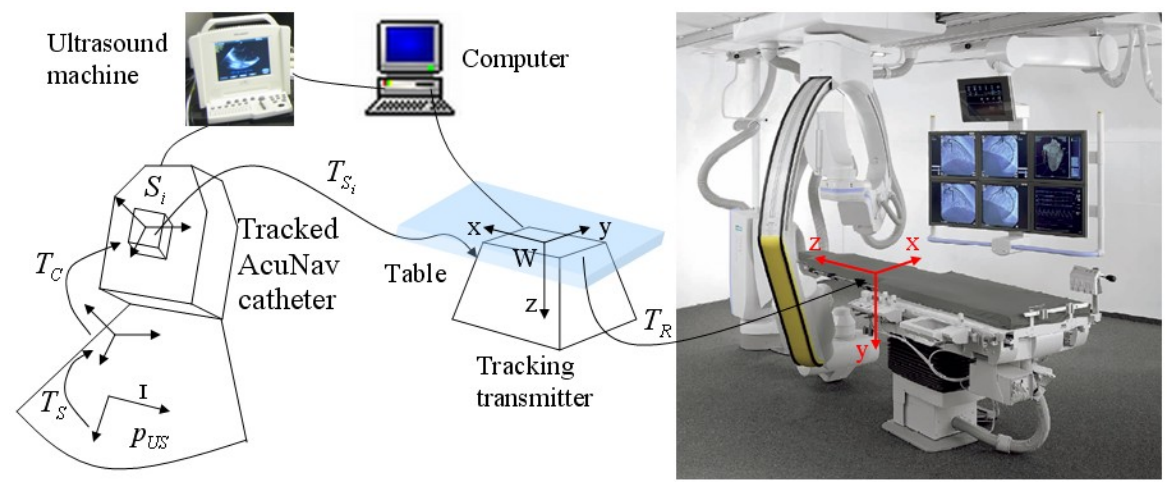

Fig. 2. System setup and transformations

them to vessel probability images. In [6, US and CT kidney images are rigidly registered. Wein et al. 7] register the ultrasound images to a CT scan on neck data of patients.

For EP applications, image integration using CartoMerge (Biosense Webster, Inc.) has been widely used, which is able to register a cloud of mapping points to the segmented surface of the pre-operative image [89. In [10], a registration between cardiac CT data and ICE is described. A point-to-surface registration, by first extracting surface point sets of the left atrium from the ICE images, is used. However, these approaches all require the segmentation of either 3D data or the ultrasound image, which itself is a challenging problem and has a direct impact on the final registration accuracy.

There are several advantages of our system compared to the existing image integration solutions to EP applications. Our image-based registration method does not require segmenting ultrasound image or CT image, so it needs little user interaction. The registration does not need the mapping of heart chambers. Mapping usually takes time and may introduce error due to respiratory motion and chamber wall deformation from the pushing of the catheter. Besides cardiac gating, we also perform respiratory gating using a position sensor, which has been ignored in previous approaches. And with C-arm CT, the doctor can access all the necessary image modalities in the operating room.

\section{Method}

\subsection{System Setup}

Figure 2 shows the set up of the system. 3D images are acquired on an Angiographic C-arm system (AXIOM Artis, Siemens Medical Solutions). To image the left atrium of a patient we acquire images during 4 consecutive rotational $190^{\circ} \mathrm{C}$-arm runs to get enough images to reconstruct a $3 \mathrm{D}$ image of one cardiac phase. Each run has 247 frames at a speed of 60 frames per second. The radiation dose is $1.2 \mu \mathrm{Gy}$ per frame. The images are reconstructed and processed 
on a PC workstation. For visualization purpose, the left atrium and other heart structures are segmented using dedicated software.

The images acquired by the ICE catheter (AcuNav, Siemens Medical Solutions) are transferred via a frame grabber card into the $\mathrm{PC}$ at 30 frames per second. To track the position of the ICE catheter tip we used a magnetic tracking system (Microbird, Ascension). Its position sensor has been integrated in the same tubing with the ultrasound transducer. The transmitter is installed under the table of the C-arm system, such that an ICE catheter above the table is within the operating range and can be tracked during an intervention. During ICE imaging we record the ECG signal of the patient, and track the position sensor at the ICE catheter tip and the other position sensor at the patient's chest (for respiratory motion correction) simultaneously.

Let us denote the coordinate system of the tracking device as $W$, and the coordinate system of the sensor when acquiring the $i$ th ICE frame as $S_{i}$. The tracking device provides transformation matrix $T_{S_{i}}$ of $S_{i}$ relative to $W$. The position of an ICE image pixel in $S_{i}$ is determined by a scaling matrix $T_{S}$ and a transformation $T_{C}$ provided by an offline calibration using the method described in 11. An ICE image pixel $\boldsymbol{p}_{\mathrm{US}}$ is mapped to a point in CT coordinate system by $\boldsymbol{p}_{\mathrm{CT}}=T_{R} \cdot T_{S_{i}} \cdot T_{C} \cdot T_{S} \cdot \boldsymbol{p}_{\mathrm{US}}$, where $T_{R}$ is the registration matrix to be estimated, as showed in Figure 2 .

\subsection{Cardiac and Respiration Motion Gating}

Heart motion as well as respiratory motion are factors that influence the accuracy of registration. To eliminate the respiratory motion from the frames to be registered, the displacement in the $\mathrm{Z}$ axis of the second sensor placed on the patient's chest was analyzed. We pick the frames acquired without significant chest movement. To detect the respiratory rest phases, the position variance of the sensor in the $\mathrm{Z}$ axis during the previous 50 acquired frames is computed. If the variance is below a given threshold, the frames would be considered. The threshold can be adjusted during the ICE image acquisition. We further select the frames with a fixed time interval from the previous R-wave in the ECG signal for cardiac gating. Typically we get about 3 cardiac gated frames for each respiration. The doctor puts the ICE catheter in the right atrium and sweeps the ICE across the heart chambers. When a qualified frame is selected, the system notifies the doctor to proceed to another orientation or position.

\subsection{Image Registration}

The registration process computes a rigid transformation from the coordinate system of the tracking device to the C-arm CT space. Because the ultrasound only images the heart and the CT image contains the whole thorax of the subject, an initial alignment between them is needed before applying the automatic search for the six registration parameters. Since the tracking device is fixed under the table, an initial rotation can be estimated from the axes of the tracking 


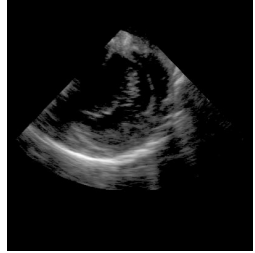

(a)

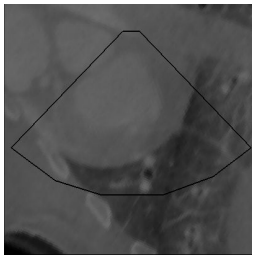

(b)

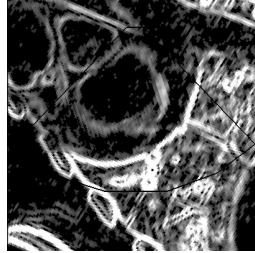

(c)

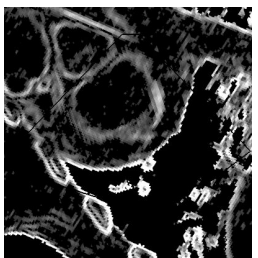

(d)

Fig. 3. Preprocessing of C-arm CT data to match ICE image. (a) 2D ICE image, (b) C-arm CT MPR image, (c) gradient image of (b), (d) after applying a mask on (c) for pixels whose intensity in (b) is higher than a threshold.

transmitter and the patient orientation of the C-arm CT data. An initial translation can be estimated by asking the user to either select a point in the 3D data corresponding to the tip location, or manually match the ICE frame and Multiplanar Reconstruction (MPR) of the 3D data. This estimation does not need to be very accurate. For example, if the segmentation of the C-arm CT data is available, the centroid of the right atrium segmentation can be used to initialize the registration.

The initial registration is followed by an automatic registration step. The registration parameters are optimized according to the similarity between the gated ICE images and its corresponding CT slices. Because ICE has high intensity at the chamber boundary and low intensity values inside the chamber, we use the gradient magnitude of the $\mathrm{CT}$ data in similarity computation. The gradient at each voxel of the CT volume is computed using 3D Sobel filter before applying the registration. Figure $3(\mathrm{a})$ to $3(\mathrm{c})$ show an ICE image, the corresponding CT slice, and the gradient magnitude on the $\mathrm{CT}$ slice. The high gradient magnitude outside the heart chamber can affect the accuracy of registration results because it does not appear in the ICE image. Therefore, a thresholding step is performed on the CT intensity image and the obtained mask is then applied to the gradient magnitude image, as showed in Figure $3(\mathrm{~d})$, This threshold value can be adjusted interactively from the user interface.

We compute the Normalized Cross-Correlation (NCC) for each pair of the ICE image and the CT gradient magnitude slice. The sum of all NCC values is taken as the similarity measure, Similarity $\left(T_{R}\right)=\sum_{i=1}^{N} \operatorname{NCC}\left(\operatorname{US}_{i}, \mathrm{CT}_{i}\left(T_{R}\right)\right)$, where $\mathrm{US}_{i}$ is the $i$ th ICE image from the gated sequence of $\mathrm{N}$ images, $\mathrm{CT}_{i}\left(T_{R}\right)$, is the resliced plane from the cardiac C-arm CT gradient magnitude data corresponding to $\mathrm{US}_{i}$ and a given transformation $T_{R}$. We take the measure only for those pixels located inside the pre-defined fan shape of the ultrasound image.

We use the best neighbor method in the optimization. An initial step size is first chosen. In each iteration, all of the parameters are changed in turn with the step size and the value of similarity measure is computed. The change that causes the greatest improvement in the similarity measure is kept. This iteration continues until no change can give a better similarity measure. Then, the step size is reduced and above process is repeated again. We choose an initial step 
size $5.5 \mathrm{~mm}$ or 5.5 degree. They are then reduced by a factor of 2 when needed. The minimum step size for stop criterion is $0.1 \mathrm{~mm}$ or 0.1 degree. We also tested other optimization strategies such as Powell method and gradient decent method. However, we get the best result using the best neighbor method. This may be due to the existence of many local optima which makes the one dimensional search and gradient estimation, used in Powell and gradient decent methods, respectively, behave poorly.

\section{Experimental Results}

During the experiment on an anesthetized pig, 54 ICE sequences were saved while the physician was imaging the pig's heart chambers. The registration was performed offline. The size of C-arm CT volume is $512 \times 512 \times 444$. The data is downsampled by a factor of 2 on each dimension to speed up the registration process. From one of the image sequences, 18 frames were selected after cardiac and respiratory gating. The ICE images were captured in a resolution of $640 \times 480$. They are clipped and resized to $128 \times 128$ for the registration. Figure 4 shows the registration results in a side by side comparison with the corresponding C-arm CT MPR images. The registration procedure implemented in $\mathrm{C}++$ took less than a minute on a system with an $2.8 \mathrm{GHz}$ Intel $\mathrm{P} 4$ processor and 2 GB DDR memory.

The registration results were validated quantitatively by comparing chamber wall distance between 2D ICE image and 3D C-arm CT images. 29 ICE images covering the LA and LV were manually selected and segmented by an ultrasound image specialist using dedicated software. Figure 5(a) shows the LA segmentation on one ICE image. Four heart chambers in 3D C-arm CT image were segmented using a tool developed for cardiac CT data. The mask boundary of the 3D segmentations cut by the ICE image plane is displayed on the ICE image, as showed in Figure 5(b). We computed the shortest distance from each pixel on the 2D segmentation boundary to the 3D segmentation boundary for all 29 ICE images. The average distance is $3.14 \pm 3.13 \mathrm{~mm}$.

As another qualitative evaluation, we constructed a 3D ultrasound volume from a dense ICE sweeping and compared it with the registered 3D C-arm CT volume. Figure $5(\mathrm{c})$ and $5(\mathrm{~d})$ show a side by side comparison of two volumes cut by the same clip plane, where the LA and LV matches well in two volumes.

Figure 6(a) shows a 2D ICE image of LA and the corresponding MPR slice of the C-arm CT image. In Figure 6(b), the ICE catheter is displayed in 3D showing the position and orientation of the ultrasound fan relatively to the segmented heart chambers. The ultrasound fan clipped the LA and the pulmonary veins can be clearly observed. Other tracked sensors can be displayed in the same coordinate system after the registration. For example, the red ball represents the location of the sensor on the chest. We also provide an endoscopic visualization of pre-operative data by setting up a perspective camera on the catheter tip, as showed in Figure 6(b) A clip plane is enabled to make the user directly see the region of interest. 


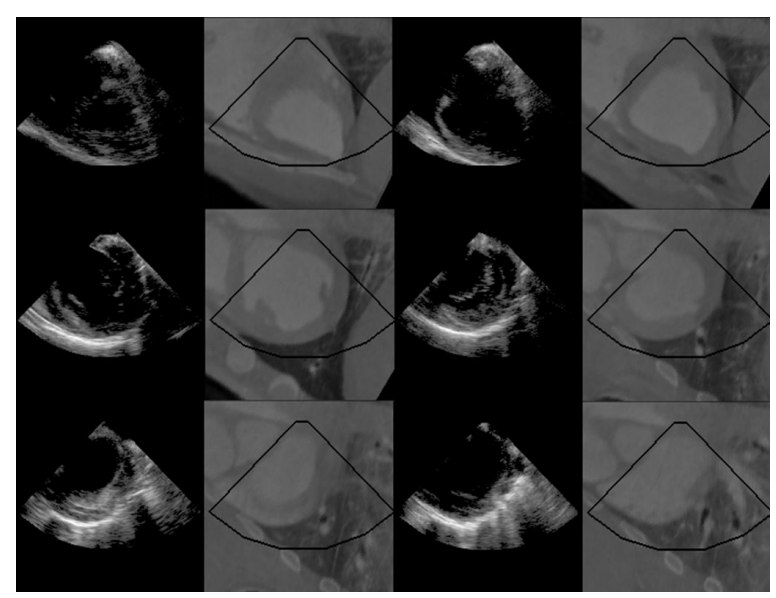

Fig. 4. A side by side comparison of the registered ICE images with C-arm CT MPR images

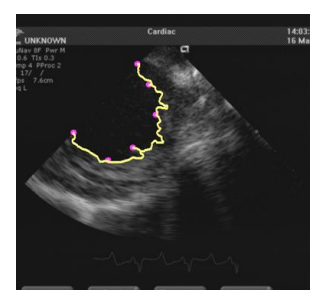

(a)

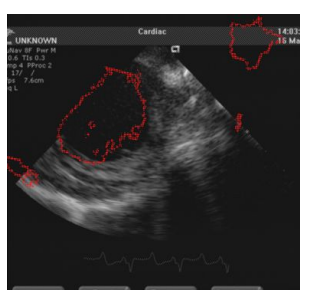

(b)

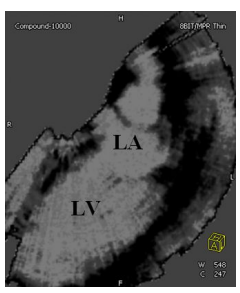

(c)

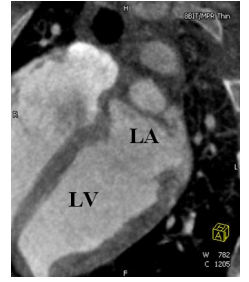

(d)

Fig. 5. Visual inspection of registration results. (a) 2D ICE image LA segmentation (yellow contour), (b) C-arm CT LA segmentation cut by the ICE image (red contour), (c) a cross-sectional image of the dense ICE acquisitions, (d) corresponding MPR of C-arm CT image after registration.

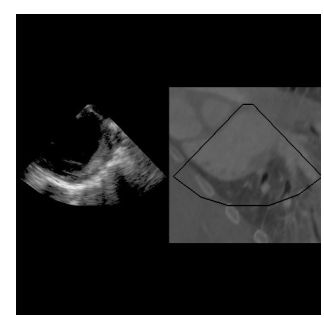

(a)

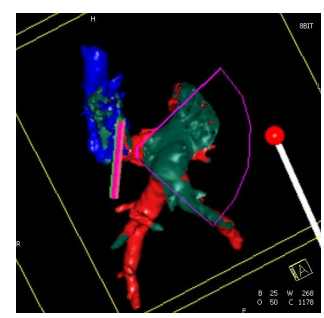

(b)

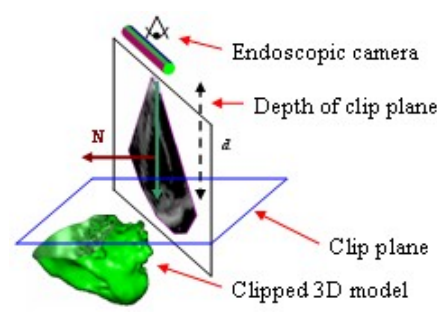

(c)

Fig. 6. (a) CT slice provides a bigger field of view on the anatomy, (b) ICE catheter and other tracked sensor (red ball) are displayed with 3D segmented heart chambers, (c) endoscopic view set up 


\section{Discussion and Future Work}

We presented a method of registering ICE images and 3D C-arm CT data for EP applications. This framework also works with other pre-operative data such as CT and MR. Compared to previous works, our method performs both cardiac and respiratory gatings. And it does not require segmenting 3D data or ICE images. We showed good registration result on data acquired in animal experiments. Currently, the method still needs user interactions to choose proper threshold and provide initial alignment before applying the registration. Our next step is to make the registration process simpler and faster and validated by physicians in online case studies. It is also possible to estimate the respiratory motion from the position sensor on the ICE catheter instead of using an extra sensor on the patient chest. We believe the proposed method can help the physicians to learn and perform complex EP ablation procedures, improve the ablation accuracy, and reduce the EP procedure time.

\section{References}

1. Pappone, C., et al.: Atrial electroanatomic remodeling after circumferential radiofrequency pulmonary vein ablation: Efficacy of an anatomic approach in a large cohort of patients with atrial fibrillation. Circulation 104, 2539-2544 (2001)

2. Maloney, J., Burnett, J., Dala-Krishna, P., Glueck, R.: New directions in intracardiac echocardiography. Journal of Interventional Cardiac Electrophysiology 13, 23-29 (2005)

3. Lauritsch, G., Boese, J., Wigstrom, L., Kemeth, H., Fahrig, R.: Towards cardiac C-arm computed tomography. IEEE Trans. Med. Imaging 25(7), 922-934 (2006)

4. Roche, A., Pennec, X., Malandain, G., Ayache, N.: Rigid registration of 3D ultrasound with MR images: a new approach combining intensity and gradient information. IEEE Trans. Med. Imaging 20(10), 1038-1049 (2001)

5. Penney, G., Blackall, J., Hamady, M., Sabharwal, T., Adam, A., Hawkes, D.: Registration of freehand 3D ultrasound and magnetic resonance liver images. Med. Image. Anal. 8(1), 81-91 (2004)

6. Leroy, A.: Rigid registration of freehand 3D ultrasound and CT-scan kidney images. In: Proc. Medical Image Computing and Computer-Assisted Intervention, pp. 837844 (2004)

7. Wein, W., Roper, B., Navab, N.: Automatic registration and fusion of ultrasound with CT for radiotherapy. In: Proc. Medical Image Computing and ComputerAssisted Intervention, pp. 303-311 (2005)

8. Sun, Y., et al.: Registration of high-resolution 3D atrial images with electroanatomical cardiac mapping: Evaluation of registration method. Proc. SPIE Medical Imaging 5744, 299-307 (2005)

9. Fahmy, T., et al.: Intracardiac echo-guided image integration. Journal of Cardiovascular Electrophysiology 18(3), 276-282 (2007)

10. Zhong, H., Kanade, T., Schwartzman, D.: Virtual Touch.: An efficient registration method for catheter navigation in left atrium. In: Proc. Medical Image Computing and Computer-Assisted Intervention, pp. 437-444 (2006)

11. Khamene, A., Sauer, F.: A novel phantom-less spatial and temporal ultrasound calibration method. In: Proc. Medical Image Computing and Computer-Assisted Intervention, pp. 65-72 (2005) 